\title{
High Serum Levels of Serum 100 Beta Protein, Neuron-specific Enolase, Tau, Active Caspase-3, M30 and M65 in Children with Autism Spectrum Disorders
}

\author{
Hamza Ayaydın', Adnan Kirmit', Hakim Çelik ${ }^{3}$, İsmail Akaltun ${ }^{4}$, İsmail Koyuncu', Şermin Bilgen Ulgar ${ }^{1}$ \\ Departments of ${ }^{1}$ Child and Adolescent Psychiatry, ${ }^{2}$ Biochemistry, and ${ }^{3}$ Physiology, Faculty of Medicine, Harran University, Şanlıurfa, ${ }^{4}$ Department \\ of Child and Adolescent Psychiatry, Gaziantep Dr. Ersin Arslan Training and Research Hospital, Gaziantep, Turkey
}

\begin{abstract}
Objective: The purpose of this study was therefore to investigate whether neuronal, axonal, and glial cell markers (Neuron-specific enolase [NSE], tau, serum 100 beta protein [S100B], respectively) and apoptosis markers (active caspase 3, M30, M65) and whether these parameters can be used as diagnostic biomarkers in autism spectrum disorders (ASD).

Methods: This study measured the serum S100B, NSE, tau, active caspase 3, M30, and M65 levels in 43 patients with ASD (aged 3-12 years) and in 41 age- and sex-matched healthy controls. ASD severity was rated using the Childhood Autism Rating Scale. The serum levels were determined in the biochemistry laboratory using the ELISA technique. The receiver operator characteristics curve method was employed to evaluate the accuracy of the parameters in diagnosing ASD.

Results: Serum S100B, tau, NSE, active caspase-3, M30, and M65 levels were significantly higher in the patient group than in the control group $(p<0.001, p=0.002, p=0.002, p=0.005, p<0.001$, and $p=0.004$, respectively). The cut-off value of S100B was $48.085 \mathrm{pg} / \mathrm{ml}$ (sensitivity: $74.4 \%$, specificity: $80.5 \%$, areas under the curve: 0.879 , $p<0.001)$.

Conclusion: Apoptosis increased in children with ASD, and neuronal, axonal, and glial cell injury was observed. In addition, S100B may be an important diagnostic biomarker in patients with ASD. Apoptosis, and neuronal, axonal and astrocyte pathologies may play a significant role in the pathogenesis of ASD, and further studies are now required to confirm this.
\end{abstract}

KEY WORDS: Autism spectrum disorder; Serum 100 beta protein; Neuron-specific enolase; Tau protein; Apoptosis; Caspase-3.

\section{INTRODUCTION}

Autism spectrum disorder (ASD) is a neurodevelopmental condition. A significant rise in the prevalence of the condition in recent years has increased the importance of clarifying its pathogenesis. Genetic factors occupy an important place in the etiology, and oxidative stress, inflammation, mitochondrial dysfunction, immune

Received: November 18, 2019 / Revised: December 24, 2019

Accepted: January 14, 2020

Address for correspondence: Hamza Ayaydın

Department of Child and Adolescent Psychiatry, Faculty of

Medicine, Harran University, Osmanbey Yerleşkesi,

Şanlıurfa-Mardin Karayolu Üzzeri 18.Km, Haliliye 63290,

Şanlıurfa, Turkey

E-mail: drhamzaayaydin@yahoo.com

ORCID: https://orcid.org/0000-0003-4909-0070 dysregulation and environmental factors have also been linked to ASD [1]. Diagnosis of ASD is based on clinical evaluation, and no biochemical diagnostic methods are currently available. It is therefore difficult to determine the risk of the disorder developing and/or to identify it before the onset of symptoms. Due to the importance of early diagnosis and educational therapies in terms of positive prognosis, identification before the onset of symptoms is crucial. Although antioxidant enzyme levels, oxidative stress markers, and cytokines have been investigated as peripheral biomarkers of ASD, no specific biomarker has to date been identified $[2,3]$.

Tau protein plays a particularly important role in the stabilization of axonal microtubules in central neurons and in the maintenance of axonal transport [4]. Aggregation

(ㄷ) This is an Open-Access article distributed under the terms of the Creative Commons Attribution Non-Commercial License (http://creativecommons.org/licenses/by-nc/4.0) which permits unrestricted non-commercial use, distribution, and reproduction in any medium, provided the original work is properly cited. 
occurs in case of hyperphosphorylation, and intraneuronal accumulation may emerge, thus leading to axonal transport impairment, synaptic miscommunication, and neurodegeneration [5,6]. Additionally, in the presence of axonal injury tau passes into the extracellular space, and from there into cerebrospinal fluid [7]. Amyloid beta peptide, which plays an important role in Alzheimer's disease, has been shown to require Tau protein to impair hippocampal synaptic plasticity [8]. In addition, candidate gene studies concerning ASD have reported that proteins involved in synaptic mechanisms are linked to ASD risk genes [9]. One study showed increased expression of cathepsin D (a lysosomal protease), responsible for the formation of tau fragments predisposed to abnormal phosphorylation, in patients with ASD [10]. Moreover, alteration has been determined in the phosphorylated:unphosphorylated tau ratio, suggestive of abnormal microtubule depolymerization, in the hippocampus of autistic-like mouse strain C58/J [11]. Lower serum tau protein levels have also been determined in an autistic group compared to a control group [12]. Evaluation of the relation between Tau protein and ASD is particularly important considering that synaptic and myelin dysfunction is present in the pathogenesis of ASD, and that microtubules and microtubule-associated proteins are important regulators of myelination, synaptic formation, and neuron production [13].

Serum 100 beta protein (S100B), which also plays a role in Tau protein phosphorylation [14], may be present at low levels in the blood of healthy individuals, but serum levels have been shown to increase in primary and secondary cerebral injury $[15,16]$. The effect of $\mathrm{S} 100 \mathrm{~B}$ is dose-dependent. At low, nanomolar, concentrations, it exhibits a neuroprotective effect [17] and plays a role in the regeneration of injured nerves [18]. At higher, micromolar, concentrations, it increases the production of reactive oxygen species (ROS) in neurons, and induces nitric oxide-dependent death of astrocytes and neurons [19]. High serum S100B levels have also been linked to various psychiatric diseases, such as schizophrenia [20] and mood disorders [21]. Studies investigating the relation between ASD and S100B in the last decade have observed high serum [22,23] and plasma [24] S100B levels.

Neuron-specific enolase (NSE) is localized in neuron cytoplasm and peripheral neuroendocrine cells [25]. An increase in NSE levels in CSF has been shown in case of neuronal injury such as stroke, head trauma, multiple sclerosis, Alzheimer's disease, and epileptic seizure [26]. Since NSE is a cytosolic enzyme, it may only appear when the cell is injured [27], and its levels have been shown to be associated with the degree of cerebral injury [28]. Wang et al. [29] reported hypermethylation of the NSE gene in $14.5 \%$ of autistic children, as well as decreased NSE RNA expression. Higher neonatal serum NSE has also been reported in patients with ASD compared to controls [30].

Caspases are cysteine proteases found in cytoplasm and are involved in apoptopsis (programmed cell death) [31]. Cytokeratin 18 (CK-18), a cytoskeleton protein released by apoptotic cells, is broken down into M30, an apoptosis marker, with caspase activation. M65 comprises both cleaved (apoptosis) and uncleaved (necrosis) CK-18 and is used as a marker for total cell death [32]. Apoptosis is thought to play a role in the pathogenesis of neurodegenerative diseases such as Alzheimer's, Hutchinson's, Parkinson's, and amyotrophic lateral sclerosis, and also of AIDS and autoimmune diseases [33]. Apoptotic signals (such as increased cellular oxidative stress or hypoxia) deriving from inside the cell result in apoptosome. Apoptosome triggers apoptosis by activating caspase-3 [34]. Apoptosis can be determined through identification of various activations known to be apoptosis-specific (such as active caspase-3 assay) at the molecular level. The last two decades have seen an increase in studies investigating the role of apoptosis in the pathogenesis of ASD. Decreased antiapoptotic $\mathrm{Bcl}-2$ protein [35] and increased cathepsin D, caspase-3 [10] and p53 [35] levels have been shown in the cerebella of autistic subjects. Decreased Bcl-2 expression and increased cathepsin D mRNA and protein have been reported in autistic lymphoblasts, and increased apoptosis has been implicated in the pathogenesis of ASD [10]. An increase in this pathological activation in apoptosis in patients with ASD has also been potentially associated with neuroanatomic abnormalities [36].

S100B, tau and NSE levels have previously been investigated separately in patients with ASD, but not in the same patient group. Additionally, although different parameters associated with apoptosis have been investigated, there has been no previous evaluation of the markers M30 and M65 in ASD patients. Considering the presence of synapse and myelin dysfunction in the patho- 
genesis of ASD, our aim was to assess levels of tau, which is involved in myelination and synaptic formation. We also planned to examine the relationship with S100B-tau in patients diagnosed with ASD by evaluating levels of S100B that causes tau phosphorylation in the same patient group. In addition, since S100B increases ROS at high concentrations and triggers apoptosis, we also aimed to assess levels of M30, M65, and active caspase-3, associated with apoptosis, and to examine the relation between these parameters and S100-tau in patients with ASD. Additionally, we also intended to examine levels of NSE, reported at high levels in patients with neonatal autism [30], in our patient group and to examine its relations with $\mathrm{S} 100 \mathrm{~B}$, tau and apoptosis. There is also still no recognized biomarker for the early diagnosis of ASD. The purpose of this study was to examine the relation between neuronal, axonal, and glial cell injury markers and autism, and to assess whether these parameters can be used as biomarkers in the diagnosis of ASD.

\section{METHODS}

\section{Study Population}

Forty-three children aged 3-12 years (30 boys, 13 girls) presenting to our outpatient clinic between March 9, 2017 and March 29, 2018, diagnosed with ASD on the basis of Diagnostic and Statistical Manual of Mental Disorders, 5th edition [37], and meeting the inclusion criteria, were included as the study group. During this time, 65 children with ASD presented to our polyclinic for the first time, but 10 exhibiting developmental regression, 9 using medication, and three diagnosed with epilepsy were excluded from our study.

The Childhood Autism Rating Scale (CARS), the validity and reliability of whose Turkish-language version has previously been confirmed [38,39], was used to evaluated the severity of ASD symptoms. CARS consists of 15 subsections, and has a minimum possible score of 15 and a maximum possible score of 60 . Accordingly, children scoring 30-36.5 are mildly-moderately autistic, and those scoring 37-60 are severely autistic [38]. The sociodemographic data form elicited information concerning first name and surname, age, sex, and parents' ages. Patients were evaluated by pediatric neurology and pediatric metabolism specialists in terms of secondary autism, and magnetic resonance imaging of the brain and electroencephalography were also performed in the context of the study.

Exclusion criteria in the patient group were any comorbid psychological disorder during evaluation (except intellectual disability), use of any medication (including vitamins and antioxidant medication) within the previous two weeks, presence of acute (such as infection (body temperature was assessed during history-taking and examination, and complete blood counts were performed) or chronic medical disease, and history of alcohol or substance use during pregnancy of mother.

The control group consisted of 41 healthy age- and sex-matched children (10 female, 31 male) admitted to the Pediatrics Department, Healthy Child Follow-up Clinic during the study period. These children in the control group underwent physical and psychiatric examinations. Children with no psychiatric and neurological, metabolic, or endocrine disease or infections, no known genetic syndromes or allergic reactions, and not using any medications were enrolled. History of maternal alcohol and substance use during pregnancy was also adopted as an exclusion criterion.

\section{Ethics}

The study was approved by the Harran University Medical Faculty Ethics Committee (session No. 03, decision No. 31, dated 09-03-2017), and was also supported by the Scientific Research Coordination Office (date 08-12-2017, protocol No. 17187).

All parents or legal guardians of the participating children gave written informed consent prior to inclusion in the study, in accordance with the Declaration of Helsinki as amended by the World Medical Association Declaration of Helsinki.

\section{Blood Specimen Collection and Biochemical Analysis}

Five milliliters of blood was collected from the antecubital vein following at least 8-hours fasting from the children comprising the patient and control groups, and was placed into gel tubes. These were immediately centrifuged at 4,000 rpm for 10 minutes at $4^{\circ} \mathrm{C}$. The serum part was separated and divided into two parts, which were then stored in Eppendorf tubes at $-80^{\circ} \mathrm{C}$ until analysis. S100B, NSE, tau, M30, M65 and active caspase-3 levels in sera were measured using the Enzyme-Linked Immunosorbent Assay (ELISA) method in the biochemistry labo- 
ratory. Specimens were read at $450 \mathrm{~nm}$ on a microplate reader (Multiskan GO; Thermo Fisher Scientific, Waltham, MA, USA). Human NSE was studied using a Fine Test ELISA kit (Catalogue No. [CN]: EH0370). NSE levels were expressed as ng/ml. Human S100B was studies using a Fine Test ELISA kit (CN. EH0543). S100B levels were expressed as $\mathrm{pg} / \mathrm{ml}$. Human tau was also measured using a Fine Test ELISA kit (CN. EH2030). Tau levels were expressed as pg/ml. Human M30 (Cyotokeratin 18-M30) was studied using a Fine Test ELISA kit (CN. EH4188), with the results being expressed as $\mathrm{mlU} / \mathrm{ml}$.

Human M65 (Cytokeratin 18-M65) was measured using a Fine Test ELISA kit (CN. EH2820), and levels were expressed as ng/ml. Finally, human active caspase-3 was studied using a SunRed ELISA kit (CN. 201-12-0970A), and levels were expressed as $\mathrm{pg} / \mathrm{ml}$.

\section{Statistical Analysis}

SPSS 23.0 software (IBM Co., Armonk, NY, USA) was used for statistical analysis, and categorical variables were analyzed using Pearson's chi-square test. Normality of distribution was tested using the Shapiro - Wilk normality test. Since data were not normally distributed, non-parametric tests were applied. The Mann - Whitney $U$ test was used for continuous and two-way group comparisons, and data were expressed as median values (interquartile range). Spearman's correlation analysis was applied to determine correlations. The receiver operator characteristics (ROC) curve method was employed to evaluate the accuracy of S100B, NSE, tau, M30, M65 and active caspase- 3 in diagnosing ASD. The ROC was plot- ted to determine cut-off points. $p$ values $<0.05$ were regarded as statistically significant.

\section{RESULTS}

Median ages were six years in the patient group (30 male, 13 female) and seven years in the control group (31 male, 10 female). No statistically significant difference was determined between the groups in terms of age, gender, parents' ages $(p>0.05)$ (Table 1). CARS scores were mild-moderate in $12(27.9 \%)$ patients and severe in 31 (72.1\%). The median CARS score in the ASD group was 41 (interquartile range: 8.5 ). Serum S100B, tau, NSE, active caspase-3, M30, and M65 levels were significantly higher in the patient group than in the control group $(p<$ 0.001, $p=0.002, p=0.002, p=0.005, p<0.001$, and $p=$ 0.004 , respectively) (Table 1 ).

Spearman' rho correlation analysis determined no significant relation between CARS score and S100B, tau, NSE, active caspase-3, M30, and M65 ( $p>0.05)$. Significant inverse correlation was determined between age of the patients and M65 levels $(r=-0.455, p=$ 0.002), but none with other parameters $(p>0.05)$. Significant positive correlation was observed between tau levels and NSE and S100B levels $(r=0.597, p<0.001$; and $r=0.433, p=0.004$, respectively). Significant positive correlation was observed between NSE levels and M65 and S100B levels $(r=0.445, p=0.003$; and $r=0.561 p<$ 0.001 , respectively). M65 levels were significantly positively correlated with $\mathrm{S} 100 \mathrm{~B}$ levels $(r=0.507, p=0.001$ ). Significant positive correlation was also determined be-

Table 1. Sociodemographic characteristics and S100B, tau, NSE, active caspase-3, M30, and M65 serum levels of the autism spectrum disorder and control groups

\begin{tabular}{|c|c|c|c|c|c|}
\hline Parameters & Patients $(n=43)$ & Controls $(n=41)$ & $p$ value & $\chi^{2}$ & Z \\
\hline Sex, F/M ${ }^{a}$ & $13(30.2) / 30(69.8)$ & $10(24.4) / 31(75.6)$ & 0.548 & 0.360 & \\
\hline Age $(y r)^{b}$ & $6(3)$ & $7(3)$ & 0.217 & & -1.235 \\
\hline Mother's age $(y r)^{b}$ & $34(11)$ & $32(8)$ & 0.713 & & -0.368 \\
\hline Father's age $(y r)^{b}$ & $37(11)$ & $37(6)$ & 0.527 & & -0.633 \\
\hline $\mathrm{S} 100 \mathrm{~B}(\mathrm{pg} / \mathrm{ml})^{\mathrm{b}}$ & $49.13(2.12)$ & $24.33(32.64)$ & $<0.001$ & & -5.979 \\
\hline Tau $(p g / m l)^{b}$ & $131.4(183.2)$ & $57.5(47)$ & 0.002 & & -5.979 \\
\hline NSE $(\mathrm{ng} / \mathrm{ml})^{\mathrm{b}}$ & $3.04(1.15)$ & $2.65(0.42)$ & 0.002 & & -3.043 \\
\hline Active caspase $3(\mathrm{pg} / \mathrm{ml})^{b}$ & $543.2(403.5)$ & $415(225.5)$ & 0.005 & & -2.837 \\
\hline $\mathrm{M} 30(\mathrm{mlU} / \mathrm{ml})^{\mathrm{b}}$ & $19.37(8.36)$ & $16.22(1.21)$ & $<0.001$ & & -4.484 \\
\hline M65 (ng/ml) & $0.30(1.55)$ & $0.20(0.11)$ & 0.004 & & -2.854 \\
\hline
\end{tabular}

Values are presented as median (interquartile range).

NSE, neuron-specific enolase; $S 100 B$, serum 100 beta protein; $F$, female; $M$, male.

${ }^{\mathrm{a}}$ Chi-square $p$ value, ${ }^{\mathrm{b}}$ Mann - Whitney $U p$ value. 
tween S100B and M30 levels ( $r=0.489, p=0.001)$.

ROC analysis was performed to determine cut-off values for the study parameters for the prediction of ASD patients. Areas under the curve (AUC) were calculated, and cut-off levels were determined as demonstrated in Figure 1. The cut-off value of parameters including tau, NSE, M65, S100B, M30 and active caspase-3 were 72.55 pg/ml (sensitivity: 69.8\%, specificity: 70.7\%, AUC: 0.697, $p=0.002$ ), $2.83 \mathrm{ng} / \mathrm{ml}$ (sensitivity: $60.5 \%$, specificity: 78\%, AUC: 0.693, $p=0.002), 0.215 \mathrm{ng} / \mathrm{ml}$ (sensitivity: 69.8\%, specificity: 65.9\%, AUC: 0.681, $p=0.004)$, $48.085 \mathrm{pg} / \mathrm{ml}$ (sensitivity: $74.4 \%$, specificity: $80.5 \%$, AUC: 0.879, $p<0.001$ ), $17.41 \mathrm{mlU} / \mathrm{ml}$ (sensitivity: 69.8\%, specificity: $80.5 \%$, AUC: $0.784, p<0.001$ ), and $456.4 \mathrm{pg} / \mathrm{ml}$ (sensitivity: $65.1 \%$, specificity: $65.9 \%$, AUC: $0.680, p=0.005)$, respectively. High S100B values indicated ASD (Fig. 1).

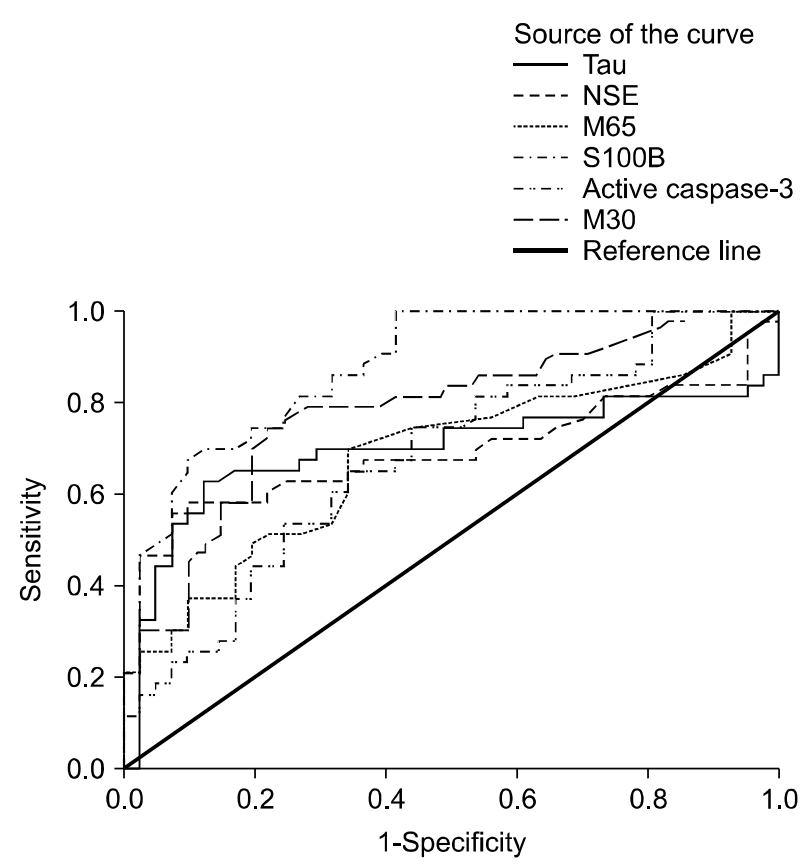

Fig. 1. Receiver operator characteristics (ROC) curves of active caspase 3, serum 100 beta protein (S100B), tau, M30, M65, neuron-specific enolase (NSE) for the prediction of autism spectrum disorders.

S100B ROC curve: area under the curve (AUC) was determined as 0.879 for S100B with a cut-off value of 48.085. Tau ROC curve: AUC was determined as 0.697 for tau with a cut-off value of 72.55 . NSE ROC curve: AUC was determined as 0.693 for NSE with a cut-off value of 2.83. M65 ROC curve: AUC was determined as 0.681 for M65 with a cut-off value of 0.215. M30 ROC curve: AUC was determined as 0.784 for M30 with a cut-off value of 17.41. Active caspase 3 ROC curve: AUC was determined as 0.680 for active caspase 3 with a cut-off value of 456.4 .

\section{DISCUSSION}

This study evaluated serum S100B, tau, NSE, active caspase-3, M30, and M65 levels in children with ASD, together with their sensitivity and specificity. The main finding of this study indicated an increase in serum S100B, tau, NSE, active caspase-3, M30, and M65 levels in children with ASD compared to the control subjects. In addition, the increased S100B level exhibited $80.5 \%$ sensitivity and $74.4 \%$ specificity according to ROC analysis. S100B has been reported to be neuroprotective at low concentrations and to play a role in the regeneration of injured neurons $[17,18]$, although at high concentrations it increases the production of reactive oxygen species in neurons and mediates the death of astrocytes and neurons [19]. High blood S100B levels have even been linked to brain damage, and have been described as a predictor of poor prognosis by themselves in traumatic brain injury [40]. Additionally, high serum S100B levels have been determined in schizophrenia [20] and mood disorders [21]. A study of the identification of risk genes for ASD through copy number variation analysis in Austrian families identified S100B as one of the genes potentially associated with ASD [41]. In agreement with our own research, two recent studies showed higher serum S100B levels in children with ASD compared to control subjects [22,23]. Al-Ayadhi and Mostafa [22] determined positive correlation between serum S100B and severity of ASD, while Shaker et al. [23] observed no correlation, in agreement with our own study. Another study reported a high plasma S100B concentration in children with ASD [24], as well as positive correlation between both S100B levels and tumor necrosis factor-alpha and severity of ASD. A decreased serum glutathione (GSH)/oxidized glutathione (GSSG) redox ratio has also been shown in patients with ASD [42]. Increased peripheral oxidative stress and malondialdehyde (lipid peroxidation marker) levels [43,44] have also been reported in children with ASD. The fact that at high concentrations $\mathrm{S} 100 \mathrm{~B}$ increases reactive oxygen species [19], and the high serum S100B values consistent with our own study $[22,23]$, suggest that S100B may be associated with the pathogenesis of ASD.

S100B exhibited positive correlation with tau, M30 and M65 levels in the present study. Under healthy conditions, S100B modulates the cell cycle (such as proliferation, differentiation, and apoptosis) in the central 
nervous system, as well as interacting with tau to promote neurite outgrowth $[45,46]$. However, increased S100B levels lead to tau hyperphosphorylation, resulting in the formation of neurofibrillary tau tangles [47]. Additionally, considering the role of $\mathrm{S} 100 \mathrm{~B}$ in apoptosis, the positive correlation with M65 and M30, apoptotic cell products, in our patient group, was another important finding suggesting that $\mathrm{S100B}$ may be involved in the pathogenesis of ASD.

Kadak et al. [12] determined lower serum $\alpha$-Synuclein and tau protein levels in children with ASD than in a control group. In contrast, in the present study, serum tau levels were higher in the ASD group than in the controls. Positive correlation was also determined between tau and S100B and NSE. Microtubules play a vital role in brain development, dendritic spine plasticity, myelination, synaptic formation, and the migration of developing neurons to their destinations [13]. The phosphorylated:unphosphorylated tau ratio in the brain is in balance. One study reported alterations in the phosphorylation pattern of different tau isoforms in the hippocampus, prefrontal cortex and cerebellum of the autistic-like mice strain C58/J, suggesting that alterations in tau regulation vary between different regions of the brain [11]. The decrease in the phosphorylated:unphosphorylated tau ratio in the hippocampus in that previous study supports the idea of abnormal microtubule depolymerization [11]. Moreover, since risk gene proteins associated with ASD are found in synaptic mechanisms [9], and different tau levels have been reported in patients with ASD and controls, tau may also be an important factor in the pathogenesis of ASD.

NSE is one of the important peripheral blood markers of neuronal injury [48]. One study reported hypermethylation of the enolase gene (ENO2) in 14.5\% (number: 19/131) in patients with ASD, together with $70 \%$ less ENO2 RNA expression compared to a control group [29]. Another previous study reported significantly higher neonatal levels of NSE in ASD patients compared to healthy controls [30]. Consistent with that study, NSE levels were also higher in our ASD group. We also determined positive correlation between NSE and tau and M65 levels. The presence of positive correlation between tau, which rises in case of axonal injury, and NSE, which rises in the event of neuronal injury, suggested that neuronal and axonal injury may play an important role in the pathogenesis of ASD.
Caspases are essential proteins with a key function in the apoptotic process. Caspase- 3 is the most important caspase in the effector phase of apoptosis [49]. Levels of the apoptotic marker M30 and of the total cell death marker M65 increase with caspase activation [32]. Impairment of balance in apoptosis plays an important role in the pathogenesis of several major diseases, such as Alzheimer's, parkinson's and autoimmune diseases [33]. Studies have also investigated the role of apoptosis in ASD with central and peripheral sampling [10,35,50-52].

Studies of postmortem brain tissue in patients with ASD have reported that $\mathrm{Bcl}-2$ decreased by $34 \%-51 \%$ in the autistic cerebellum [50], 38\% in the autistic superior frontal cortex, and $36 \%$ in the autistic cerebellum [52]. Another study determined low Bcl-2 levels and high expression of the Bcl-2 regulator p53 in Purkinje cells and granule cells [35]. Studies have reported 130\%, 67.5\%, and $38 \%$ increases in p53 levels in the autistic parietal, superior frontal, and cerebellar cortices, respectively, while the $\mathrm{Bcl}-2 / \mathrm{P} 53$ ratio decreased by $64 \%$ in the autistic brain compared to control subjects [50]. Increased cathepsin D, associated with tau cleavage, and also caspase-3 have also been shown in the autistic cerebellum [10]. In addition, a review of postmortem studies of the brains of autistic patients reported cerebellar Purkinje cell atrophy, a decrease in numbers, and granule cell loss [36]. Pathological activation of apoptosis may therefore be associated with neuroanatomical changes and ASD. Another study determined lower plasma caspase-3 in children with ASD compared to control subjects [53]. The low plasma caspase-3 level in that study may have derived from the conversion of caspase-3 into active caspase-3. Indeed, the other study showed up-regulation and activation of several caspases, together with increases in levels of caspase-3, -7, and -12 in peripheral blood mononuclear cells of children with ASD [54]. Similarly to the increased apoptosis in children with ASD described above, we also observed higher active caspase-3, M30 and M65 levels in our ASD group compared to the control group. Evaluation of this increase in serum active caspase-3 and M30 levels in our study together with the decrease in Bcl-2 [50] and the increase in p53 [35] shown in the brain tissue of individuals with ASD suggested that apoptosis increases in children with ASD. In addition, evaluation of the increase in M65 (the total cell death marker) levels in our study together with the atrophy in 
and loss of cerebellar cells and the low number of those cells [36] suggested that apoptosis and necrosis increase in neuronal cells in children with ASD. Indeed, significant inverse correlation between age and serum M65 levels in patients diagnosed with ASD suggested that apoptosis and necrosis in neuron and glial cells was more intense in younger children. Additionally, M30 and M65 exhibiting positive correlation with S100B, NSE, and M65 suggests an association with the apoptotic process in neuronal and astrocyte cells, and that injury in these cells may be associated with the pathogenesis of ASD.

Another important finding of this report was elicited by the ROC curve analysis applied to assess the diagnostic value of increased S100B, tau, NSE, active caspase-3, M30, and M65 parameters. Although recent studies have investigated pro-oxidant and antioxidant markers of potential diagnostic value in ASD, there is still no generally accepted biomarker for diagnosing and determining the severity of ASD $[2,3]$. The majority of the markers evaluated in the present study have not previously been investigated as diagnostic biomarkers in children with ASD. Only one study has evaluated a low plasma caspase level (AUC: 0.968) as a diagnostic biomarker in children with ASD [53]. In contrast to that study, we assessed active caspase-3 levels and determined significant elevation in children with ASD. The high active caspase-3 level, one finding from our study, and the low caspase-3 level shown in the previous study [53] suggest that apoptosis increases in children with ASD. ROC analysis applied in order to evaluate active caspase-3 in children with ASD (sensitivity: 65.1\%, specificity: $65.9 \%$, AUC: 0.680), revealed no diagnostic value. ROC analysis also revealed no diagnostic value in terms of ASD for tau (sensitivity: $69.8 \%$, specificity: $70.7 \%$, AUC: 0.697 ), NSE (sensitivity: $60.5 \%$, specificity: 78\%, AUC: 0.693), M65 (sensitivity: 69.8\%, specificity: 65.9\%, AUC: 0.681 ) or M30 (sensitivity: 69.8\%, specificity: $80.5 \%$, AUC: 0.784). In addition, ROC analysis identified S100B (sensitivity: $74.4 \%$, specificity: 80.5\%, AUC: 0.879 ) as having good diagnostic value in children with ASD.

Limitations of the present study include the low sample number, and the fact that parameters were studied in serum, not in cerebrospinal fluid. The diagnostic value of these parameters in children with ASD should now be evaluated in further studies with more extensive samples. In conclusion, our study indicated an increase in serum
S100B, tau, NSE, active caspase-3, M30, and M65 levels in children with ASD. In addition, positive correlation was determined between S100B and tau, M30, and M65 levels, between tau and NSE, and between NSE and M65 levels. Inverse correlation was determined between patient age and M65. These findings suggest the presence of neuronal, axonal, and glial cell injury in children diagnosed with ASD, and that apoptosis and necrosis increase and may be more intense in younger children in particular. This also supports the importance of early diagnosis and intervention in autism. In addition, our findings suggest that S100B may be a promising, potential biomarker for ASD and worthy of further study.

\section{- Acknowledgments}

We would like to thank the patients and their families for allowing us to publish this article. This research was supported by the Harran University Scientific Research Coordination Office (protocol No. 17187, dated 08/12/2017).

\section{- Conflicts of Interest}

No potential conflict of interest relevant to this article was reported.

\section{Author Contributions}

Conceptualization: Hamza Ayaydın, İsmail Koyuncu. Data acquisition: Hamza Ayaydın, Şermin Bilgen Ulgar, İsmail Akaltun, Adnan Kirmit. Formal analysis: Hamza Ayaydın, Adnan Kirmit, İsmail Koyuncu, Hakim Çelik, Şermin Bilgen Ulgar, İsmail Akaltun. Supervision: Hamza Ayaydın. Writing - original draft: Hamza Ayaydın, İsmail Koyuncu, Hakim Çelik. Writing - review \& editing: Hamza Ayaydın, İsmail Koyuncu, Adnan Kirmit.

\section{- ORCID}

Hamza Ayaydın https://orcid.org/0000-0003-4909-0070 Adnan Kirmit https://orcid.org/0000-0003-2799-8416

Hakim Çelik https://orcid.org/0000-0002-7565-3394

İsmail Akaltun https://orcid.org/0000-0002-9938-9276

İsmail Koyuncu https://orcid.org/0000-0002-9469-4757 Şermin Bilgen Ulgar

https://orcid.org/0000-0001-5237-220X

\section{REFERENCES}

1. Rossignol DA, Frye RE. A review of research trends in physiological abnormalities in autism spectrum disorders: immune 
dysregulation, inflammation, oxidative stress, mitochondrial dysfunction and environmental toxicant exposures. Mol Psychiatry 2012;17:389-401.

2. Khramova TV, Kaysheva AL, Ivanov YD, Pleshakova TO, lourov IY, Vorsanova SG, et al. Serologic markers of autism spectrum disorder. J Mol Neurosci 2017;62:420-429.

3. Masi A, Glozier N, Dale R, Guastella AJ. The immune system, cytokines, and biomarkers in autism spectrum disorder. Neurosci Bull 2017;33:194-204.

4. Watanabe A, Hasegawa M, Suzuki M, Takio K, MorishimaKawashima M, Titani K, et al. In vivo phosphorylation sites in fetal and adult rat tau. J Biol Chem 1993;268:25712-25717.

5. de la Monte SM. Brain insulin resistance and deficiency as therapeutic targets in Alzheimer's disease. Curr Alzheimer Res 2012;9:35-66.

6. Johnson GV, Stoothoff WH. Tau phosphorylation in neuronal cell function and dysfunction. J Cell Sci 2004;117(Pt 24): 5721-5729.

7. Shaw GJ, Jauch EC, Zemlan FP. Serum cleaved tau protein levels and clinical outcome in adult patients with closed head injury. Ann Emerg Med 2002;39:254-257.

8. Shipton OA, Leitz JR, Dworzak J, Acton CE, Tunbridge EM, Denk F, et al. Tau protein is required for amyloid \{beta\}-induced impairment of hippocampal long-term potentiation. J Neurosci 2011;31:1688-1692.

9. Parellada M, Penzol MJ, Pina L, Moreno C, González-Vioque E, Zalsman G, et al. The neurobiology of autism spectrum disorders. Eur Psychiatry 2014;29:11-19.

10. Sheikh AM, Li X, Wen G, Tauqeer Z, Brown WT, Malik M. Cathepsin D and apoptosis related proteins are elevated in the brain of autistic subjects. Neuroscience 2010;165:363-370.

11. Barón-Mendoza I, García O, Calvo-Ochoa E, Rebollar-García JO, Garzón-Cortés D, Haro R, et al. Alterations in neuronal cytoskeletal and astrocytic proteins content in the brain of the autistic-like mouse strain C58/J. Neurosci Lett 2018;682:32-38.

12. Kadak MT, Cetin I, Tarakçıoğlu MC, Özer ÖF, Kaçar S, Çimen B. Low serum level $\alpha$-synuclein and tau protein in autism spectrum disorder compared to controls. Neuropediatrics 2015;46:410-415.

13. Chang Q, Yang H, Wang M, Wei H, Hu F. Role of microtubule-associated protein in autism spectrum disorder. Neurosci Bull 2018;34:1119-1126.

14. Baudier J, Cole RD. Interactions between the microtubule-associated tau proteins and S100b regulate tau phosphorylation by the $\mathrm{Ca}^{2+} /$ calmodulin-dependent protein kinase II. J Biol Chem 1988;263:5876-5883.

15. Shirasaki Y, Edo N, Sato T. Serum S-100b protein as a biomarker for the assessment of neuroprotectants. Brain Res 2004;1021:159-166.

16. Wunderlich MT. Head injury outcome prediction in the emergency department: a role for protein S-100B? J Neurol Neurosurg Psychiatry 2003;74:827-828.

17. Van Eldik LJ, Christie-Pope B, Bolin LM, Shooter EM, Whetsell
WO Jr. Neurotrophic activity of S-100 beta in cultures of dorsal root ganglia from embryonic chick and fetal rat. Brain Res 1991;542:280-285.

18. Bhattacharyya A, Oppenheim RW, Prevette D, Moore BW, Brackenbury R, Ratner N. S100 is present in developing chicken neurons and Schwann cells and promotes motor neuron survival in vivo. J Neurobiol 1992;23:451-466.

19. Michetti F, Corvino V, Geloso MC, Lattanzi W, Bernardini C, Serpero $\mathrm{L}$, et al. The $\mathrm{S} 100 \mathrm{~B}$ protein in biological fluids: more than a lifelong biomarker of brain distress. J Neurochem 2012; 120:644-659.

20. Zhang XY, Xiu MH, Song C, Chen DC, Wu GY, Haile CN, et al. Increased serum S10OB in never-medicated and medicated schizophrenic patients. J Psychiatr Res 2010;44:12361240.

21. Schroeter ML, Sacher J, Steiner J, Schoenknecht P, Mueller K. Serum $S 100 B$ represents a new biomarker for mood disorders. Curr Drug Targets 2013;14:1237-1248.

22. Al-Ayadhi LY, Mostafa GA. A lack of association between elevated serum levels of $S 10 O B$ protein and autoimmunity in autistic children. I Neuroinflammation 2012;9:54.

23. Shaker NM, Taha GRA, Kholeif H, Sayed NM, El-Sheikh MM, Abulmagd ML. Serum levels of S100b, interleukin-6 and anti-transg/utaminase li IgA as immune markers in a sample of Egyptian children with autistic spectrum disorders. Autism Open Access 2016;6:1000191.

24. Guloksuz SA, Abali O, Aktas Cetin E, Bilgic Gazioglu S, Deniz $\mathrm{G}$, Yildirim A, et al. Elevated plasma concentrations of $\mathrm{S} 100$ calcium-binding protein $B$ and tumor necrosis factor alpha in children with autism spectrum disorders. Rev Bras Psiquiatr 2017;39:195-200.

25. Heshmati HM, Touitou Y, Foubert L, Young WF Jr, Bruckert E, Turpin G. Serum neuron-specific enolase in patients with pituitary adenoma. Clin Chem 1997;43:540-541.

26. Royds JA, Davies-Jones GA, Lewtas NA, Timperley WR, Taylor CB. Enolase isoenzymes in the cerebrospinal fluid of patients with diseases of the nervous system. J Neurol Neurosurg Psychiatry 1983;46:1031-1036.

27. Johnsson P, Blomquist S, Lührs C, Malmkvist G, Alling C, Solem JO, et al. Neuron-specific enolase increases in plasma during and immediately after extracorporeal circulation. Ann Thorac Surg 2000;69:750-754.

28. Song TJ, Choi YC, Lee KY, Kim WJ. Serum and cerebrospinal fluid neuron-specific enolase for diagnosis of tuberculous meningitis. Yonsei Med J 2012;53:1068-1072.

29. Wang Y, Fang Y, Zhang F, Xu M, Zhang J, Yan J, et al. Hypermethylation of the enolase gene (ENO2) in autism. Eur J Pediatr 2014;173:1233-1244.

30. Lv MN, Zhang H, Shu Y, Chen S, Hu YY, Zhou M. The neonatal levels of TSB, NSE and CK-BB in autism spectrum disorder from Southern China. Transl Neurosci 2016;7:6-11.

31. Bantel H, Ruck P, Gregor M, Schulze-Osthoff K. Detection of elevated caspase activation and early apoptosis in liver dis- 
eases. Eur J Cell Biol 2001;80:230-239.

32. Woolbright BL, Bridges BW, Dunn W, Olson JC, Weinman $\mathrm{SA}$, Jaeschke $\mathrm{H}$. Cell death and prognosis of mortality in alcoholic hepatitis patients using plasma keratin-18. Gene Expr 2017; 17:301-312.

33. Thompson CB. Apoptosis in the pathogenesis and treatment of disease. Science 1995;267:1456-1462.

34. Afford S, Randhawa S. Apoptosis. Mol Pathol 2000;53:55-63.

35. Sheikh AM, Malik M, Wen G, Chauhan A, Chauhan V, Gong $C X$, et al. BDNF-Akt-BCl2 antiapoptotic signaling pathway is compromised in the brain of autistic subjects. J Neurosci Res 2010;88:2641-2647.

36. Malik M, Sheikh AM, Wen G, Spivack W, Brown WT, Li X. Expression of inflammatory cytokines, BCl2 and cathepsin D are altered in lymphoblasts of autistic subjects. Immunobiology 2011;216:80-85.

37. Wei H, Alberts I, Li X. The apoptotic perspective of autism. Int J Dev Neurosci 2014;36:13-18.

38. American Psychiatric Association. DSM-5 Task Force. Diagnostic and statistical manual of mental disorders: DSM-5. Arlington, VA:American Psychiatric Association;2013.

39. İncekaş Gassaloğlu S, Baykara B, Avcil S, Demiral Y. [Validity and reliability analysis of Turkish version of childhood autism rating scale]. Turk Psikiyatri Derg 2016;27:266-274. Turkish.

40. Schopler E, Reichler RJ, DeVellis RF, Daly K. Toward objective classification of childhood autism: Childhood Autism Rating Scale (CARS). J Autism Dev Disord 1980;10:91-103.

41. Di Battista AP, Buonora JE, Rhind SG, Hutchison MG, Baker AJ, Rizoli SB, et al. Blood biomarkers in moderate-to-severe traumatic brain injury: potential utility of a multi-marker approach in characterizing outcome. Front Neurol 2015;6:110.

42. Egger G, Roetzer KM, Noor A, Lionel AC, Mahmood H, Schwarzbraun $\mathrm{T}$, et al. Identification of risk genes for autism spectrum disorder through copy number variation analysis in Austrian families. Neurogenetics 2014;15:117-127.

43. Han Y, Xi QQ, Dai W, Yang SH, Gao L, Su YY, et al. Abnormal transsulfuration metabolism and reduced antioxidant capacity in Chinese children with autism spectrum disorders. Int
J Dev Neurosci 2015;46:27-32.

44. Zoroglu SS, Armutcu F, Ozen S, Gurel A, Sivasli E, Yetkin O, et al. Increased oxidative stress and altered activities of erythrocyte free radical scavenging enzymes in autism. Eur Arch Psychiatry Clin Neurosci 2004;254:143-147.

45. Altun $\mathrm{H}$, Şahin N, Kurutaş EB, Karaaslan U, Sevgen FH, Findıklı E. Assessment of malondialdehyde levels, superoxide dismutase, and catalase activity in children with autism spectrum disorders. Psychiatr Clin Psychopharmacol 2018;28: 408-415.

46. Donato R, Sorci G, Riuzzi F, Arcuri C, Bianchi R, Brozzi F, et al. S100B's double life: intracellular regulator and extracellular signal. Biochim Biophys Acta 2009;1793:1008-1022.

47. Kawata K, Liu CY, Merkel SF, Ramirez SH, Tierney RT, Langford D. Blood biomarkers for brain injury: what are we measuring? Neurosci Biobehav Rev 2016;68:460-473.

48. Esposito G, Scuderi C, Lu J, Savani C, De Filippis D, luvone T, et al. S10OB induces tau protein hyperphosphorylation via Dickopff-1 up-regulation and disrupts the Wnt pathway in human neural stem cells. J Cell Mol Med 2008;12:914-927.

49. Taylor CB, Royds JA, Parsons MA, Timperley WR. Diagnostic aspects of enolase isozymes. Isozymes Curr Top Biol Med Res 1983;11:95-119.

50. Boatright KM, Salvesen GS. Mechanisms of caspase activation. Curr Opin Cell Biol 2003;15:725-731.

51. Fatemi SH, Halt AR, Stary JM, Realmuto GM, Jalali-Mousavi M. Reduction in anti-apoptotic protein BCl-2 in autistic cerebellum. Neuroreport 2001;12:929-933.

52. Fatemi SH, Stary JM, Halt AR, Realmuto GR. Dysregulation of Reelin and BCl-2 proteins in autistic cerebellum. J Autism Dev Disord 2001;31:529-535.

53. Araghi-Niknam M, Fatemi SH. Levels of BCl-2 and P53 are altered in superior frontal and cerebellar cortices of autistic subjects. Cell Mol Neurobiol 2003;23:945-952.

54. El-Ansary AK, Ben Bacha AG, Al-Ayadhi LY. Proinflammatory and proapoptotic markers in relation to mono and di-cations in plasma of autistic patients from Saudi Arabia. J Neuroinflammation 2011;8:142. 